\title{
Comparison between efficacy of Solifenacin and Oxybutynin for the treatment of overactive bladder in women.
}

\footnotetext{
1. MBBS, FCPS Urology

Registrar Urology

Institute of Kidney Diseases, Peshawar.

2. MBBS, M.Phil (Pharmacology) Assistant Professor Pharmacology Khyber Medical College.

3. MBBS, FCPS Urology

Registrar Urology

Institute of Kidney Diseases, Peshawar.

4. MBBS, FCPS Urology Assistant Professor Urology Institute of Kidney Diseases, Peshawar.

5. MBBS, FCPS Urology Registrar Urology Institute of Kidney Diseases, Peshawar.

6. MBBS

Medical Officer

Institute of Kidney Diseases, Peshawar.
}

Correspondence Address: Dr. Muhammad Saleh Faisal Assistant Professor, Pharmacology Khyber Medical College. drsalehfaisal@gmail.com

Article received on: 29/09/2020 Accepted for publication: 05/11/2020

\begin{abstract}
Jehanzeb1, Muhammad Saleh Faisal ${ }^{2}$, Samiullah Opal ${ }^{3}$, Ahmad Nawaz ${ }^{4}$, Muneeb Hassan ${ }^{5}$, Sikandar Hayat ${ }^{6}$

ABSTRACT... Objective: To compare the efficacy of solifenacin and oxybutynin in the treatment of overactive bladder in female patients. Study Design: Quasi Experimental study. Setting: Department of Urology, Institute of Kidney Diseases, Peshawar. Period: Sept 2017 to Mar 2018. Material \& Methods: After selection of 312 female patients with overactive bladder by convenience sampling, written informed consent was taken and pre-treatment overactive bladder symptoms (OABS) score was calculated. Patients were randomly distributed in two equal groups of 156 subjects, where Group A received Oxybutynin (3 mg, BD) and Group B received Solifenacin $(5 \mathrm{mg}, \mathrm{OD})$ for a period 12 weeks. Patients were called for follow up visit to determine and compare the efficacy of study drugs. Those who showed improvement in at least 03 points in OABS score taken pre-treatment were considered as positive responders. Results: The mean age of patients was $38.8 \pm 7.62$ years with age range from 20 to 50 years. $110(70.5 \%)$ subjects in oxybutynin treated group while $126(80.8 \%)$ subjects in solifenacin treated group reported significant improvement in OAB symptoms. Conclusion: Solifenacin is relatively more effective than oxybutynin for treating female patients with overactive bladder.
\end{abstract}

Key words: $\quad$ Efficacy, Oxybutynin, Overactive Bladder, Solifenacin.

Article Citation: Jehanzeb, Faisal MS, Opal S, Nawaz A, Hassan M, Hayat S. Comparison between efficacy of Solifenacin and Oxybutynin for the treatment of overactive bladder in women. Professional Med J 2021; 28(6):896-901. https://doi.org/10.29309/TPMJ/2021.28.06.6118

\section{INTRODUCTION}

Overactive bladder (OAB) is a complex of symptoms, characterized by urinary urgency with or without urge incontinence, increased urinary frequency and nocturia, in the absence of underlying pathological condition explaining these features. This definition was proposed in a standardization of terminology report by international continence society (ICS). ${ }^{1}$ Urgency is a mandatory component of this definition, describing the fact that urgency is the major driving force behind all other elements like incontinence, frequency and nocturia. ${ }^{2,3}$ These symptoms are due to over activity of detrusor muscle as demonstrated by urodynamic tests but urethro-vesical dysfunctions can also cause similar clinical features. ${ }^{4}$

Globally, millions of people are suffering from $\mathrm{OAB}$. In America, it is estimated that approximately seventeen million people are affected by this disease with increasing prevalence as age increases. Studies reported OAB prevalence of $16.5 \%$ in US population aged $\geq 18$ years and $16.6 \%$ in European individuals aged $\geq 40$ years and by the year 2025, there will be substantial rise in this number. ${ }^{5}$ The quality of life of patient is more negatively affected by OAB than by asthma, hypertension, cardiac diseases or diabetes mellitus. Even their quality of life is much poorer than the lives of people with depression. ${ }^{6}$ Several reports have been published describing the association of urinary incontinence with significant healthcare cost. In the year 2000 , estimated total economic cost associated with $\mathrm{OAB}$ was 12.6 billion dollars in USA while in Sweden, urinary incontinence alone accounts approx $2 \%$ of the total healthcare cost. ${ }^{7}$

As far as the management of OAB syndrome is concerned, various treatment options are available aiming to reduce the magnitude of 
symptoms. Anti-muscarinics are considered as the first line agents i.e. Oxybutynin, Solifenacin, Darifenacin, Propiverine, Tolterodine, Trospium, Fesoterodine and Flavoxate. They block the muscarinic receptors of detrusor muscle, reducing its activity and hence the symptoms. In our setup, Oxybutynin and Solifenacin are more commonly prescribed. Oxybutynin has been in use for over 20 years and available in different dosage forms whereas Solifenacin is an anticholinergic drug which is more bladder selective and well-tolerated. These two drugs have been assessed in various randomized controlled trials against placebo or compared with other competitors where both found to be effective in patients of $\mathrm{OAB}{ }^{8,9}$ In a clinical trial where 863 patients with $\mathrm{OAB}$ symptoms were randomized with solifenacin versus placebo, the former has shown significant reduction in the episodes of urinary urgency, with or without incontinence. ${ }^{10}$ But a meta-analysis based on 50 randomized controlled trials including more than 27000 female patients revealed only modest reduction in OAB symptoms after treatment with antimuscarinic drugs. They caused reduction in urge incontinence by 1.73 episodes/day and voids by 2.06/day whereas, placebo decreased the urge incontinence by 1.06 episodes/day and voids by $1.2 /$ day. This analysis revealed no individual drug to be superior than other competitors. ${ }^{11}$

Hence, there is a lot of disparity in the data on efficacy of anti-muscarinic drugs in terms of urgency and episodes of urge incontinence. This study was an attempt to identify more efficacious agent between two commonly used antimuscarinic drugs for OAB i.e. Oxybutynin and Solifenacin. The local data will help the clinicians in selecting most appropriate pharmacotherapy for overactive bladder in our population.

\section{MATERIAL \& METHODS}

This experimental study was carried out at Institute of Kidney Diseases (IKD), Peshawar from Sept 2017 to Mar 2018. A sample size of 312 patients was determined by WHO calculator, using $38 \%$ efficacy of oxybutynin, $52 \%$ efficacy of solifenacin, 95\% confidence level and $80 \%$ power of test. ${ }^{12}$ After taking approval from the hospital ethical committee and written informed consent, female patients visiting urology OPD fulfilling the inclusion criteria i.e. 20-50 years of age, urinary frequency of $>07$ times/day, at least 01 episode of urinary urgency/week with or without urinary incontinence, nocturia at least 01 episode/night, with duration of these symptoms more than three months and baseline OABS score of 04 were enrolled in the study. (The OABS score is a symptom assessment questionnaire designed to quantify $\mathrm{OAB}$ symptoms into a single score. ${ }^{13}$ The questionnaire consists of 4 questions on $\mathrm{OAB}$ symptoms with maximum scores ranging from 2 to 5: daytime frequency (2 points), nighttime frequency (3 points), urgency (5 points), and UUI (5 points). The total score ranges from 0 to 15 points, with higher scores indicating higher symptom severity).

To minimize the confounding and biasness in the outcome, pregnant women, patients of stress incontinence, malignancy in the pelvic organs, pelvic organ prolapse, bladder stones, UTI, neurological conditions (e.g. spinal injury, multiple sclerosis, parkinson disease, diabetic neuropathy) and medical conditions contraindicating the use of study drugs were excluded from the study. After detailed history, physical examination and calculating pretreatment OABS score, necessary investigations like Ultrasound abdomen and pelvis, Post void residual urine volume (PVRV), retrograde urethrogram, Micturating cystourethrogram (MCUG) and MRI were performed where needed. Patients were randomly allocated two groups (156 in each) where Group A received Oxybutynin (3mg, BD) and Group B received Solifenacin (5mg, OD) for a period of 12 weeks. They were called for follow up visit to determine the drug efficacy by observing significant difference in OABS score before and after treatment. Patients showing an improvement in at least 03 points in pre-treatment OABS score was considered positive responders. All the relevant data was recorded in a predesigned proforma.

\section{Data Analysis}

The collected data was analyzed in SPSS version 20. Numerical variables like age and duration 
of symptoms were described as Mean \pm SD. Categorical variables like efficacy were described in terms of frequency and percentages. Chisquare test was applied to compare the efficacy of oxybutynin and solifenacin among different groups, keeping $p$ value $<0.05$ as significant. Efficacy was stratified against age and duration of OAB symptoms to see effect modification.

\section{RESULTS}

A total of 312 female patients of overactive bladder comprising of age range from 20 to 50 years, with mean age of $38.8 \pm 7.62$ years were observed. In oxybutynin treated group, $10(6.4 \%)$ patients were of age $\leq 25$ years, $70(44.9 \%)$ patients were in age range of $26-40$ years and $76(48.7 \%)$ patients were of age $>40$ years with mean age $38.5 \pm$ 7.78 years. In solifenacin treated group, $4(2.6 \%)$ patients were of age $\leq 25$ years, 66(42.3\%) patients were in age range of $26-40$ years and $86(55.1 \%)$ patients were of age $>40$ years with mean age $39.3 \pm 7.47$ years. The distribution of age between two groups was statistically insignificant with $p$ value 0.191. (Figure-1).

In oxybutynin treated group, the average duration of symptoms was $6.9 \pm 2.11$ days with $12(7.7 \%)$ patients having duration $\leq 2$ days, $18(11.5 \%)$ patients having $3-5$ days and $126(80.8 \%)$ patients having symptoms for $>5$ days. While in solifenacin treated group, the average duration of symptoms was $7 \pm 2.23$ days with $18(11.5 \%)$ patients having duration $\leq 2$ days, 11(7.1\%) patients having $3-5$ days and $127(81.4 \%)$ patients having symptoms for $>5$ days. This distribution of duration of symptoms between two groups was also statistically insignificant with $p$ value 0.235 . (Figure-1)

Distribution of efficacy between two groups is demonstrated in Figure-2, where oxybutynin was found to be effective in $110(70.5 \%)$ patients while solifenacin in 126(80.8\%) patients. The response of both drugs was statistically significant with $p$ value 0.024 .

Age wise distribution of efficacy in both groups revealed efficacy greater in younger patients which decreased with increasing age i.e. among patients of age $\leq 25$ years, oxybutynin showed efficacy in $90 \%$ subjects and solifenacin in $75 \%$ subjects whereas among patients of age $>40$ years, oxybutynin showed efficacy in $69.7 \%$ subjects and solifenacin in $61.6 \%$ subjects. This age wise distribution of efficacy between two groups was statistically insignificant with $p$ value $>0.05$. When efficacy was stratified over duration of symptoms in both groups, the results were insignificant except for duration of symptoms $>5$ days where oxybutynin reported greater efficacy than solifenacin. (Table-I)

\section{DISCUSSION}

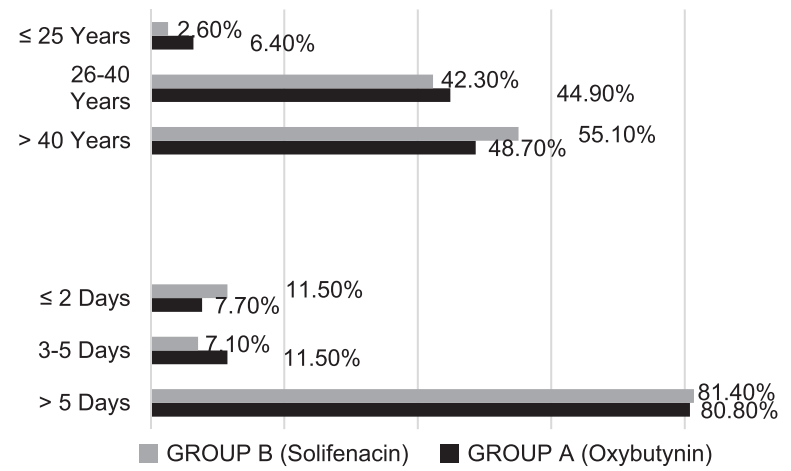

Figure-I. Distribution of age and duration of symptoms between two groups

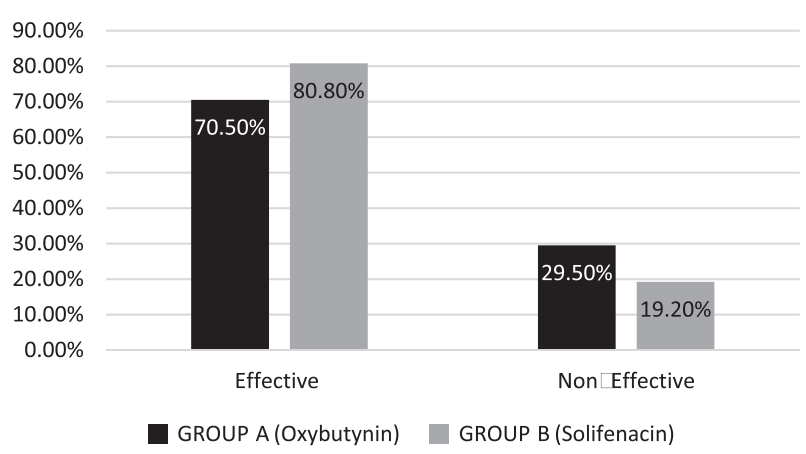

Figure-2. Distribution of efficacy between two groups

Overactive bladder is a common deplorable urological condition, poorly affecting the quality of life of many patients. ${ }^{14}$ While managing the patients of overactive bladder syndrome, antimuscarinic agents are considered as mainstay of treatment with aim to maintain the bladder's conservative and retaining functions. 


\begin{tabular}{|c|c|c|c|c|}
\hline \multicolumn{2}{|l|}{ AGE } & Group A & Group B & P-Value \\
\hline \multirow{2}{*}{$\leq 25$ years } & Effective & $9(90 \%)$ & $3(75 \%)$ & \multirow{3}{*}{0.5055} \\
\hline & Not Effective & $1(10 \%)$ & $1(25 \%)$ & \\
\hline Total & & $10(6.4 \%)$ & $4(2.6 \%)$ & \\
\hline \multirow{2}{*}{$26-40$ years } & Effective & $64(91.4 \%)$ & $54(81.8 \%)$ & \multirow{3}{*}{0.0804} \\
\hline & Not Effective & $6(8.6 \%)$ & $12(18.2 \%)$ & \\
\hline Total & & $70(44.9 \%)$ & $66(42.3 \%)$ & \\
\hline \multirow{2}{*}{$>40$ years } & Effective & $53(69.7 \%)$ & $53(61.6 \%)$ & \multirow{3}{*}{0.1795} \\
\hline & Not Effective & $23(30.3 \%)$ & $33(38.4 \%)$ & \\
\hline Total & & $76(48.7 \%)$ & $86(55.1 \%)$ & \\
\hline \multicolumn{2}{|c|}{ Duration of Symptoms } & Group A & Group B & P-Value \\
\hline \multirow{2}{*}{$\leq 2$ Days } & Effective & $11(91.7 \%)$ & $13(72.2 \%)$ & \multirow{3}{*}{0.2044} \\
\hline & Not Effective & 1 (8.3\%) & $5(27.8 \%)$ & \\
\hline Total & & $12(7.7 \%)$ & $18(11.5 \%)$ & \\
\hline \multirow{2}{*}{ 3-5 Days } & Effective & $14(77.8 \%)$ & $11(100 \%)$ & \multirow{3}{*}{0.1288} \\
\hline & Not Effective & $4(22.2 \%)$ & $0(0 \%)$ & \\
\hline Total & & $18(11.5 \%)$ & $11(7.1 \%)$ & \\
\hline \multirow{2}{*}{$>5$ Days } & Effective & $101(80.2 \%)$ & $86(67.7 \%)$ & \multirow{3}{*}{0.0171} \\
\hline & Not Effective & $25(19.8 \%)$ & $41(32.3 \%)$ & \\
\hline Total & & $126(80.8 \%)$ & $127(81.4 \%)$ & \\
\hline
\end{tabular}

Various anti-muscarinic drugs such as tolterodine, solifenacin, oxybutynin, trospium chloride, propiverine and fesoterodine have been widely used since long due to their proven efficacy, tolerability and stability. ${ }^{15,16}$ The basic mechanism of action of these drugs is antagonism of muscarinic (M3) receptors located in the smooth muscles of bladder. Though, anti-muscarinics are commonly in use for treating $\mathrm{OAB}$ patients but which drug is more effective, is not yet clear. ${ }^{17}$

Many studies have been done previously regarding $\mathrm{OAB}$ prevalence in women with reported value of $16.9 \%$ which increased with advancement in age i.e. $4.8 \%$ in women with age $<25$ years, rising to $30.9 \%$ in those with age $>65$ years. ${ }^{18}$ Another epidemiological survey done in Europe revealed $\mathrm{OAB}$ prevalence of $16.6 \%$ in a population of age $\geq 40$ years and again was found to increase with age. ${ }^{19}$ These findings resemble the results of our study where only $6.4 \%$ and $2.6 \%$ OAB cases were of age $\leq 25$ years in oxybutynin and solifenacin treated groups, respectively but increased to $48.7 \%$ and $55.1 \%$ in those having age more than 40 years.
As far as efficacy is concerned, in this study solifenacin 5mg OD appeared to be relatively more efficacious than oxybutynin, resembling the results of trials done previously revealing solifenacin $5 \mathrm{mg}$ OD as drug of choice for treating OAB symptoms. Moreover, if $5 \mathrm{mg}$ OD solifenacin fails as primary drug, then $3 \mathrm{mg}$ TDS oxybutynin need to be considered as it offers efficacy similar to $10 \mathrm{mg}$ OD solifenacin. ${ }^{20}$ There are various studies including randomized controlled trials which justified the superiority of solifenacin when compared with placebo and other anti-muscarinic drugs accounting its safety, clinical efficacy and cost. $^{21}$

In contrast, there are reports which denies the superiority of solifenacin over oxybutynin. Kakar et al compared oxybutynin, solifenacin, fesoterodine and tolterodine with a conclusion that while tolterodine and solifenacin were equally effective for treating $\mathrm{OAB}$, the oxybutynin and solifenacin showed improved efficacy, but more adverse effects were also reported. ${ }^{22}$ In clinical trials, differences in the efficacy of various anti-muscarinic agents have often reached to 
statistical significance. However in routine clinical practice, the magnitude of these differences are not readily appreciable and many clinicians labeled the efficacy of these drugs "comparable".

In this study, some shortcomings which are worth to mention includes lack of data regarding tolerability of the study drugs and assessment of drug responses at various doses.

\section{CONCLUSION}

It is concluded that solifenacin at a dose of $5 \mathrm{mg}$ OD appears to be slightly more favorable than oxybutynin at a dose of $3 \mathrm{mg}$ BD for the treatment of overactive bladder in women.

\section{Copyright@ 05 Nov, 2020.}

\section{REFERENCES}

1. Leron E, Weintraub AY, Mastrolia SA, Schwarzman P. Overactive bladder syndrome: Evaluation and management. Current Urology. 2017; 11(3):117-25.

2. Peyronnet B, Mironska E, Chapple C, Cardozo L, Oelke $M$, Dmochowski R, et al. A comprehensive review of overactive bladder pathophysiology: On the way to tailored treatment. European Urology. 2019; 75(6):9881000.

3. Saleem A. In women with urinary incontinence how necessary is cystometry? JPMA. 2010; 60(356).

4. Chen SL, Ng SC, Huang YH, Chen GD. Are patients with bladder oversensitivity different from those with urodynamically proven detrusor over activity in female overactive bladder syndrome? Journal of the Chinese Medical Association. 2017; 80(10):644-50.

5. Chuang YC, Liu SP, Lee KS, Liao L, Wang J, Yoo TK, et al. Prevalence of overactive bladder in China, Taiwan and South Korea: Results from a cross $\square$ sectional, population $\square$ based study. LUTS: Lower Urinary Tract Symptoms. 2019; 11(1):48-55.

6. Milsom I, Kaplan SA, Coyne KS, Sexton CC, Kopp ZS. Effect of bothersome overactive bladder symptoms on health-related quality of life, anxiety, depression, and treatment seeking in the United States: results from EpiLUTS. Urology. 2012; 80(1):90-6.

7. Powell LC, Szabo SM, Walker D, Gooch K. The economic burden of overactive bladder in the United States: A systematic literature review. Neurourology and Urodynamics. 2018; 37(4):1241-9.
8. Oefelein MG. Safety and tolerability profiles of anticholinergic agents used for the treatment of overactive bladder. Drug Safety. 2011; 34(9):733-54.

9. Kirshenbaum E. Update on the pharmacologic management of overactive bladder: The present and the future. Urologic Nursing. 2010; 30(1):29.

10. Wein AJ, Chapple C. Pharmacological treatment of overactive bladder. Overactive Bladder in Clinical Practice: Springer; 2012. p. 83-113.

11. Reynolds WS, McPheeters M, Blume J, Surawicz T, Worley $\mathrm{K}$, Wang $\mathrm{L}$, et al. Comparative effectiveness of anticholinergic therapy for overactive bladder in women: A systematic review and meta-analysis. Obstetrics \& Gynecology. 2015; 125(6):1423-32.

12. Hesch K, Editor Agents for treatment of overactive bladder: A therapeutic class review. Baylor University Medical Center Proceedings; 2015: Taylor \& Francis.

13. Hung MJ, Chou CL, Yen TW, Chuang YC, Meng E, Huang ST, et al. Development and validation of the Chinese overactive bladder symptom score for assessing overactive bladder syndrome in a RESORT study. Journal of the Formosan Medical Association. 2013; 112(5):276-82.

14. Chapple C, Sievert KD, MacDiarmid S, Khullar V, Radziszewski P, Nardo C, et al. Onabotulinumtoxin A $100 \mathrm{U}$ significantly improves all idiopathic overactive bladder symptoms and quality of life in patients with overactive bladder and urinary incontinence: $A$ randomized, double-blind, placebo-controlled trial. European Urology. 2013; 64(2):249-56.

15. Yamada S, Ito Y, Nishijima S, Kadekawa K, Sugaya K. Basic and clinical aspects of antimuscarinic agents used to treat overactive bladder. Pharmacology \& Therapeutics. 2018; 189:130-48.

16. Smith AL, Wein AJ. Antimuscarinic pharmacotherapy foroveractive bladder. ContemporaryPharmacotherapy of Overactive Bladder: Springer; 2019. p. 85-113.

17. Nambiar AK, Bosch R, Cruz F, Lemack GE, Thiruchelvam $\mathrm{N}$, Tubaro $\mathrm{A}$, et al. EAU guidelines on assessment and nonsurgical management of urinary incontinence. European Urology. 2018; 73(4):596-609.

18. Tailor VK, Digesu GA. Overactive bladder. Postmenopausal diseases and disorders: Springer; 2019. p. 133-44.

19. Eapen RS, Radomski SB. Review of the epidemiology of overactive bladder. Research and Reports in Urology. 2016; 8:71. 
20. Nalliah S, Gan PW, Masten Singh PK, Naidu P, Lim V, Ahamed AA. Comparison of efficacy and tolerability of pharmacological treatment for the overactive bladder in women: A network meta-analysis. Australian Family Physician. 2017; 46(3):139.
21. Basra $R$, Kelleher $C$. A review of solifenacin in the treatment of urinary incontinence. Therapeutics and Clinical Risk Management. 2008; 4(1):117.

22. Kakar H, Kok B, Mokhles S, Sarwar M. Comparison of festerodine, tolterodine, oxybutyinin and solifenacin in patients with overactive bladder. Erasmus $\mathrm{J}$ Med. 2012; 2(2):20-4.

\begin{tabular}{|c|c|c|c|}
\hline \multicolumn{4}{|c|}{ AUTHORSHIP AND CONTRIBUTION DECLARATION } \\
\hline Sr. \# & Author(s) Full Name & Contribution to the paper & Author(s) Signature \\
\hline 1 & Jehanzeb & Data collection. & \\
\hline 2 & Muhammad Saleh Faisal & Manuscript writing. & \\
\hline 3 & Samiullah Opal & Data collection. & \\
\hline 4 & Ahmad Nawaz & Concept and design of study. & \\
\hline 5 & Muneeb Hassan & $\begin{array}{l}\text { Statistical analysis and critical } \\
\text { review. }\end{array}$ & \\
\hline 6 & Sikandar Hayat & $\begin{array}{l}\text { Statistical analysis and critical } \\
\text { review. }\end{array}$ & \\
\hline
\end{tabular}

